\title{
On the stress integration in large strain elasto-plasticity
}

\author{
Francisco J. Montáns ${ }^{\mathrm{a}, *}$, Klaus-Jürgen Bathe ${ }^{\mathrm{b}}$ \\ ${ }^{a}$ Universidad de Castilla La Mancha, Camilo Jose Cela s/n, 13071-Ciudad Real, Spain \\ ${ }^{b}$ Massachusetts Institute of Technology, 77 Massachusetts Avenue, Cambridge, MA 02139, USA
}

\begin{abstract}
We briefly compare three algorithms for large strain elastoplastic analysis in which the logarithmic strain tensor is used to measure the deformations. The first and second algorithms are based on using, respectively, the elastic stretch matrices in the right basis and left basis of the polar decomposition of the trial elastic deformation gradient, and the third algorithm uses the left basis and the Finger deformation tensor.
\end{abstract}

Keywords: Computational plasticity; Finite strains; Logarithmic strains; Constitutive modelling

\section{Introduction}

In the last decade improved numerical solutions of continua subjected to large inelastic strains have been obtained by changing the initial hypo-elasto-plastic algorithms to procedures based on hyperelastic formulations. These elastic algorithms are energy preserving and are objective since no stress rates are present [1]. Different strain functions have been used, but those based on the logarithmic strains are of special interest. Their additive nature [2] and the use of the exponential mapping for the flow rule made possible the use of small strain algorithms for the plastic part; see Weber and Anand [3], Eterović and Bathe [4]. Similar frameworks have been used by different authors, among them we cite the works of Perić, Owen and Honnor [5], Cuitiño and Ortiz [6], and Simó [7]. In particular, Simó used the left polar decomposition and the Finger deformation tensor to obtain the logarithmic spatial strains and then performed the plastic stress correction in the principal spatial directions of the trial state.

Despite some differences among the cited works, three main frameworks may be distinguished. In the first framework, the right polar decomposition is used; in the second approach the left polar decomposition is employed and in the third framework the Finger tensor is used leading to an algorithm in which rotations are not involved in the case of isotropic hardening functions. In the following, we give a brief comparison of the algorithms. For simplicity we focus our discussion on deviatoric $J_{2}$ plasticity, but the

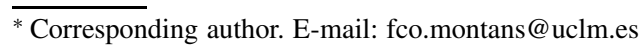

conclusions apply, in essence, also to many other material models.

\section{Constitutive frameworks}

Multiplicative plasticity is based on Lee's decomposition of the deformation gradient tensor $\mathbf{X}$ into an elastic $\mathbf{X}^{E}$ and a plastic $\mathbf{X}^{P}$ part (we use the notation of [1]):

$\mathbf{X}=\mathbf{X}^{E} \mathbf{X}^{P}$

The polar decomposition theorem establishes that for valid deformations, the elastic deformation gradient admits the following two decompositions:

$\mathbf{X}^{E}=\mathbf{R}^{E} \mathbf{U}^{E}=\mathbf{V}^{E} \mathbf{R}^{E}$

where $\mathbf{U}^{E}$ and $\mathbf{V}^{E}$ are respectively the right and left stretch tensors and $\mathbf{R}^{E}$ is the elastic rotation tensor. The Hencky (or logarithmic) elastic strain tensor and a spatial Hencky elastic strain tensor may be defined as:

$\mathbf{E}^{E}=\ln \mathbf{U}^{E} ; \quad \boldsymbol{\varepsilon}^{E}=\ln \mathbf{V}^{E}=\mathbf{R}^{E} \mathbf{E}^{E}\left(\mathbf{R}^{E}\right)^{T}$

Similarly, we define the rotated stress tensor (see $[1,3,4]$ ) as

$\overline{\mathbf{T}}=J\left(\mathbf{R}^{E}\right)^{T} \boldsymbol{\tau} \mathbf{R}^{E}$

where $\boldsymbol{\tau}$ is the Cauchy stress tensor and $J=\operatorname{det}(\mathbf{X})$ is the Jacobian determinant of the deformation gradient. For the isotropic elastic stress-strain law it can be shown that $[1,4]$ :

$J \boldsymbol{\tau}: \mathbf{D}=\overline{\mathbf{T}}: \dot{\mathbf{E}}^{E}+\overline{\mathbf{T}}: \overline{\mathbf{D}}^{P}$ 
with the following stress expressions which follow from the dissipation inequality

$\boldsymbol{\tau}=\frac{2}{J} \frac{\partial \psi}{\partial \mathbf{B}^{E}} \mathbf{B}^{E}=\frac{\partial \hat{\psi}}{\partial \boldsymbol{\varepsilon}^{E}} ; \quad \overline{\mathbf{T}}=\frac{\partial \bar{\psi}}{\partial \mathbf{E}^{E}}$

where $\mathbf{D}$ is the symmetric part of the spatial velocity gradient and $\overline{\mathbf{D}}^{P}$ is the symmetric modified plastic velocity gradient $\overline{\mathbf{L}}^{P}$ defined as $\overline{\mathbf{L}}^{P}=\dot{\mathbf{X}}^{P}\left(\mathbf{X}^{P}\right)^{-1}$. The functions $\psi, \hat{\psi}$ and $\bar{\psi}$ are the free energy functions expressed in terms of the applicable variables. These expressions define the work-conjugate quantities for an algorithm. It can be shown that in the case of associated plasticity, the principle of maximum dissipation implies:

$\mathbf{D}^{P}=-\frac{1}{2}\left(\mathcal{L}_{v} \mathbf{b}^{E}\right)\left(\mathbf{b}^{E}\right)^{-1}=\dot{\gamma} \frac{\partial f(\boldsymbol{\tau}-\boldsymbol{\beta}, \boldsymbol{\gamma})}{\partial \boldsymbol{\tau}} ;$

$\overline{\mathbf{D}}^{P}=\dot{\lambda} \frac{\partial \bar{f}(\overline{\mathbf{T}}-\overline{\mathbf{B}}, \lambda)}{\partial \overline{\mathbf{T}}}$

where $f, \bar{f}$ are the yield functions defined respectively in the spatial and the rotated descriptions, $\mathbf{b}^{E}$ is the elastic Finger tensor, $\mathcal{L}_{v}$ means the Lie derivative, $\dot{\gamma}, \dot{\lambda}$ are consistency parameters (the flow tensors are taken as unitary) and $\boldsymbol{\beta}, \overline{\mathbf{B}}$ are the back stresses in the spatial and rotated configurations respectively [4], i.e.,

$\overline{\mathbf{B}}=J\left(\mathbf{R}^{E}\right)^{T} \boldsymbol{\beta} \mathbf{R}^{E}$

We note here that the actual value of the yield function is invariant to any rigid body motion if the proper quantities are employed. It is also straightforward to show that

$\frac{\partial \bar{f}(\overline{\mathbf{T}}-\overline{\mathbf{B}}, \gamma)}{\partial \overline{\mathbf{T}}}=J\left(\mathbf{R}^{E}\right)^{T} \frac{\partial f(\boldsymbol{\tau}-\boldsymbol{\beta}, \lambda)}{\partial \boldsymbol{\tau}} \mathbf{R}^{E}$

Of course, a principal rotated configuration may be defined such that the following diagonal stress tensor is obtained [7]

$\tilde{\mathbf{T}}=J \tilde{\mathbf{R}}^{T} \boldsymbol{\tau} \tilde{\mathbf{R}}=\operatorname{Diag}\left(\tilde{T}_{1}, \tilde{T}_{2}, \tilde{T}_{3}\right)$

The previous comments are also applicable when this configuration is used, for which in a similar way, the quantities $\tilde{\mathbf{D}}^{P}, \partial \tilde{f} / \partial \tilde{\mathbf{T}}, \tilde{\mathbf{B}}$ are defined, but they are not necessarily diagonal.

\section{Numerical algorithms}

The widely used Euler backward numerical algorithms are based on the elastic-predictor plastic-corrector phases. The elastic predictor in the hyperelastic models is based directly on

$\mathbf{X}_{*}^{E}={ }_{0}^{t+\Delta t} \mathbf{X}\left({ }_{0}^{t} \mathbf{X}^{P}\right)^{-1}$

where we have used the notation of $[1,8]$. The polar decomposition of the predicted elastic deformation gradient is $\mathbf{X}_{*}^{E}=\mathbf{R}_{*}^{E} \mathbf{U}_{*}^{E}=\mathbf{V}_{*}^{E} \mathbf{R}_{*}^{E}$. Both $\mathbf{U}_{*}^{E}$ and $\mathbf{V}_{*}^{E}$ may be used to evaluate the stresses from the invariant stored elastic energy function

$$
\begin{aligned}
\psi^{E}\left(J^{E}, \boldsymbol{\varepsilon}^{\prime E}\right) & =\frac{1}{2} K\left[\ln J^{E}\right]^{2}+\mu \boldsymbol{\varepsilon}^{\prime E}: \boldsymbol{\varepsilon}^{\prime E} \equiv \bar{\psi}^{E}\left(J^{E}, \mathbf{E}^{\prime E}\right) \\
& =\frac{1}{2} K\left[\ln J^{E}\right]^{2}+\mu \mathbf{E}^{\prime E}: \mathbf{E}^{\prime E}
\end{aligned}
$$

where $K$ is the bulk modulus, $\mu$ is the shear modulus, $J^{E}=\operatorname{det}\left(\mathbf{X}^{E}\right)$ and $(\cdot)^{\prime}$ is the deviatoric part properly defined in view of Flory's decomposition. In terms of principal stretches, the stresses can also be evaluated using $\tilde{\psi}^{E}\left(J^{E}, \tilde{\mathbf{E}}^{\prime E}\right)$ where $\tilde{\mathbf{E}}^{\prime E}$ is the diagonal tensor of principal elastic stretches. It is immediate to check that the trial stress values are

$\overline{\mathbf{T}}_{*}=\frac{\partial \bar{\psi}}{\partial \mathbf{E}_{*}^{E}}=K\left(\ln _{0}^{t+\Delta t} J^{E}\right) \mathbf{1}+2 \mu \ln \mathbf{E}_{*}^{E}$

and similarly for $\boldsymbol{\tau}_{*}$ and $\tilde{\mathbf{T}}_{*}$ but in terms of $\boldsymbol{\varepsilon}_{*}^{E}$ and $\tilde{\mathbf{E}}_{*}^{E}$ respectively. We note here that [4]

$$
\begin{aligned}
& \exp \left(2 \mathbf{E}_{*}^{\prime E}\right)=\exp \left(\Delta t^{t+\Delta t} \overline{\mathbf{D}}^{P}\right) \exp \left(2{ }_{0}^{t+\Delta t} \mathbf{E}^{\prime E}\right) \exp \left(\Delta t{ }^{t+\Delta t} \overline{\mathbf{D}}^{P}\right) \\
& { }_{0}^{t+\Delta t} \mathbf{R}^{E}=\mathbf{R}_{*}^{E} \exp \left(\mathbf{E}_{*}^{\prime E}\right) \exp \left(-\Delta t^{t+\Delta t} \overline{\mathbf{D}}^{P}\right) \exp \left(-{ }_{0}^{t+\Delta t} \mathbf{E}^{\prime E}\right)
\end{aligned}
$$

which for the general case and sufficiently small time steps yields the approximations $\mathbf{E}_{*}^{\prime E} \simeq{ }^{t+\Delta t}{ }_{0} \mathbf{E}^{\prime E}+\Delta t^{t+\Delta t} \overline{\mathbf{D}}^{P}$ and ${ }_{0}^{t+\Delta t} \mathbf{R}^{E} \simeq \mathbf{R}_{*}^{E}\left(\simeq{ }_{0}^{t+\Delta t} \mathbf{R}\right)$. In the special case of pure isotropic hardening the equality holds since $\overline{\mathbf{D}}^{P}, \mathbf{E}_{*}^{\prime E}$ and ${ }^{t+\Delta t} \mathbf{E}^{\prime E}$ have the same eigenspaces. Thus, once the predictor is established, the corrector phase is applied in an almost irrotational way which allows for the use of any basis for this task. Note that using the principal rotated configuration (using Eq. 10) requires the solution of an eigenproblem [7] whereas in the other approaches this computation may be avoided through the use of proper approximations for the exponential and logarithmic functions of a tensor [3,4] and the explicit solution for the square root of a tensor [9] (all of them independent of whether the eigenvalues are equal or not). On the other hand, no advantage is obtained in kinematic hardening using the configuration of Eq. (10) since the back-stress tensor needs to be updated and the setting advocated in [7] yields different results if this task is not performed. Note that the yield stress is invariant to rotations of the quantity $\boldsymbol{\tau}-\boldsymbol{\beta}$, but not of any of the components independently as stated in Ref. [7].

A further analysis of the algorithms with tables of the actual procedures may be found in Ref. [10].

\section{Examples}

Consider a single element with prescribed displacements such that the deformation gradients are 
$\lambda$

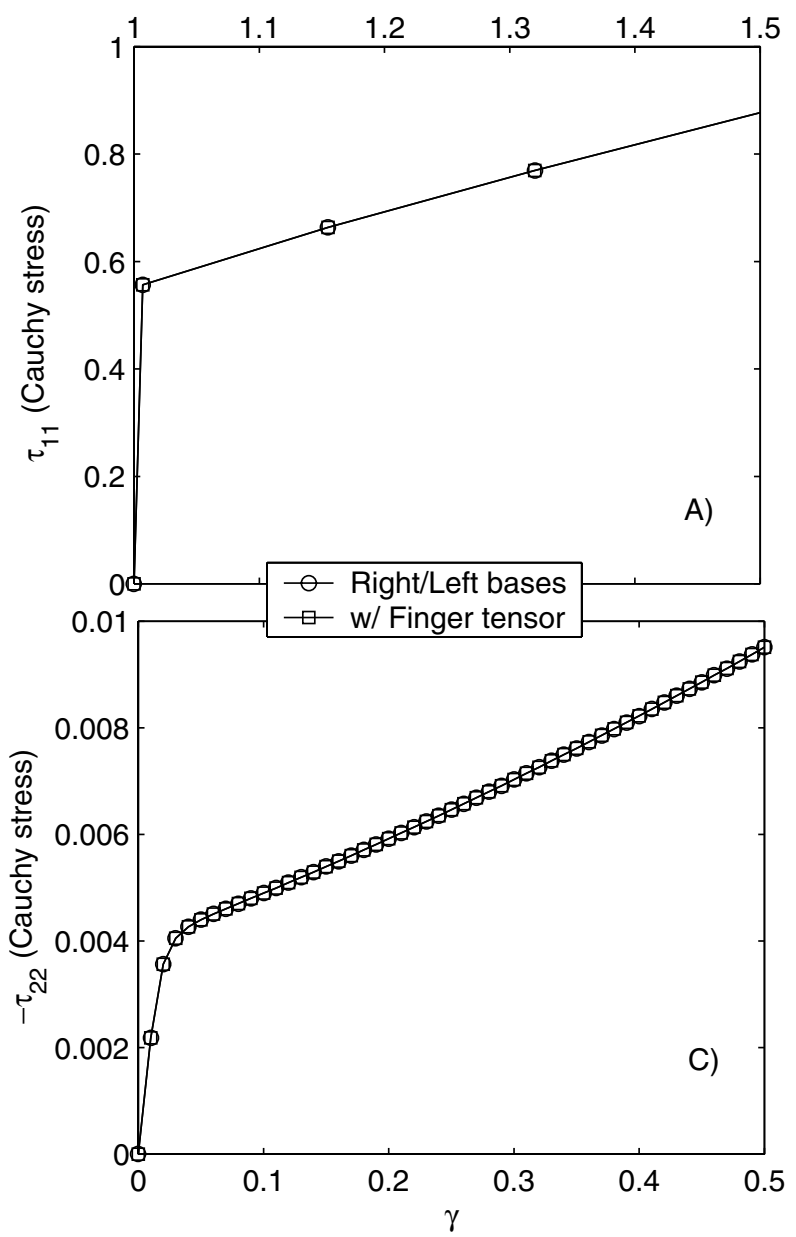

$\gamma$

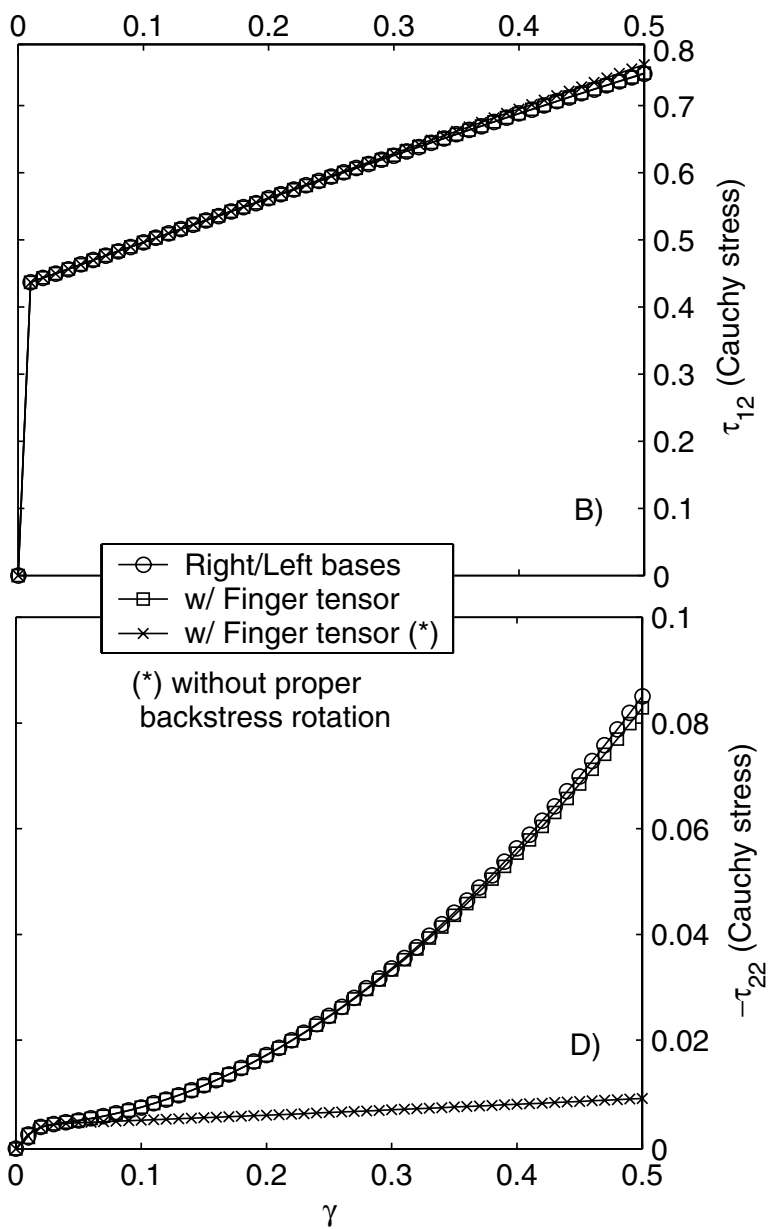

Fig. 1. Example 1: (A) stress $\tau_{11}$ vs. stretch $\lambda$ (all algorithms). Example 2: (B) $\tau_{12}$ vs. $\gamma$ for kinematic hardening (results for isotropic hardening, not shown, are virtually coincident). (C) $-\tau_{22}$ vs. $\gamma$ for isotropic hardening. (D) $-\tau_{22}$ vs. $\gamma$ for kinematic hardening.

Example 1: $\quad{ }_{0}^{t} \mathbf{X}=\left[\begin{array}{ccc}\lambda & 0 & 0 \\ 0 & 1 / \lambda & 0 \\ 0 & 0 & 1\end{array}\right]$;

Example 2: $\quad{ }_{0}^{t} \mathbf{X}=\left[\begin{array}{lll}1 & \gamma & 0 \\ 0 & 1 & 0 \\ 0 & 0 & 1\end{array}\right]$

with $\lambda=[1,1.5]$ and $\gamma=[0,0.5]$. These examples have been considered in [4] for the algorithm using the right basis. For simplicity linear hardening is considered. The material constants are: $K=166.67 \mathrm{MPa}, \mu=76.92 \mathrm{MPa}$, $\sigma_{0}=0.75 \mathrm{MPa}$ (initial yield stress), $H=2.0 \mathrm{MPa}$ (linear hardening modulus). It is seen from Fig. 1 that, as predicted from the previous arguments, the results obtained using both bases are coincident for both isotropic and kinematic hardening, and almost coincident for the algorithm using the left basis and the Finger tensor. In the case of kinematic hardening it is also seen that if the backstress is not updated to the basis used in the plastic correction, the results differ substantially in example 2 for the $\tau_{22}$ component. We refer to Reference [10] for further details and examples.

\section{Conclusions}

We briefly reviewed and compared three stress calculation algorithms for isotropic elastoplasticity considering large strains based on the multiplicative decomposition and the logarithmic strains. The stress calculations are performed in the bases of the left and the right polar decompositions of the elastic deformation gradient, whereas the plastic integration may be performed in any rotated configuration. No significant difference in the results is ob- 
tained if the proper update is performed for the backstress tensor. From the computational standpoint, the operations and cost are similar, but in the case of the algorithm using the left basis and the Finger tensor, the cost is higher due to the need for the proper rotation update of the backstress [10].

\section{Acknowledgments}

The first author wishes to acknowledge the support of the Ministerio Español de Ciencia y Tecnología through project DPI2002-02065 and that of the Junta de Comunidades de Castilla La Mancha. Also, partial financial support for the stay of the first author at MIT was provided by the Universidad de Castilla La Mancha.

\section{References}

[1] Bathe KJ. Finite Element Procedures. Englewood Cliffs, NJ: Prentice Hall, 1996.

[2] Seth BR. Generalized strain measure with applications to physical problems. In: Reiner M, Abir D (Eds), SecondOrder Effects in Elasticity, Plasticity, and Fluid Dynamics. Oxford: Pergamon Press, 1964, 2, pp. 162-172.
[3] Weber G, Anand L. Finite deformation constitutive equations and a time integration procedure for isotropic hyperelastic-viscoplastic solids. Comput Methods Appl Mech Eng 1990;79:173-202.

[4] Eterović A, Bathe KJ. A hyperelastic-based large strain elasto-plastic constitutive formulation with combined isotropic-kinematic hardening using the logarithmic stress and strain measures. Int J Numer Methods Eng 1990;30:1099_ 1114.

[5] Perić D, Owen DJR, Honnor ME. A model for finite strain elasto-plasticity based on logarithmic strains: Computational issues. Comput Methods Appl Mech Eng 1992; 94:35-61.

[6] Cuitiño A, Ortiz M. A material-independent method for extending stress update algorithms from small strain plasticity to finite plasticity with multiplicative kinematics. Eng Comput 1992;9:437-451.

[7] Simó JC. Algorithms for static and dynamic multiplicative plasticity that preserve the classical return mapping schemes of the infinitesimal theory. Comput Methods Appl Mech Eng 1992;99:61-112.

[8] Kojić M, Bathe KJ. Inelastic Analysis of Solids and Structures, in preparation. New York: Springer-Verlag, 2003.

[9] Hoger A, Carlson DE. Determination of the stretch and rotation in the polar decomposition of the deformation gradient. Q Appl Math 1984;42:113-117.

[10] Montáns FJ, Bathe KJ. Computational issues in large strain elasto-plasticity (in preparation). 